\title{
Vasomotor Center Controls of the Hepatic Circulation during Blood Pressure Oscillation in Rabbits
}

\author{
Toru Takeuchi, Nobuyuki Terada, and Shozo Koyama* \\ Department of Physiology, Yamanashi Medical College, \\ Tamaho, Yamanashi, 409-38 Japan \\ *Department of Physiology, Kagawa Medical School, \\ Miki, Kagawa, 761-07 Japan
}

\begin{abstract}
Changes in hepatic hemodynamics during blood pressure oscillations caused by the side pressure exertion procedure (SPEP) were studied in anesthetized rabbits. The brain was excluded from systemic circulation except for one of the common carotids, which was compressed with stepwise elevated pressure. In normovolemia, the hepatic arterial flow (HAF) increased as the systemic arterial pressure (SAP) rose up to $140 \mathrm{mmHg}$, and then decreased as SAP rose further. With the rise of SAP; the portal venous flow (PVF) initially rose slightly, followed by a nearly constant decrease. Hemorrhaging influenced slightly the changes of HAF and PVF with the SAP rise. After volume loading, SAP and PVF markedly increased but HAF changed little. However, the initial increase in HAF with the SAP rise was markedly depressed by volume loading. PVF conspicuously decreased without initial increase with the rise in SAP. The hepatic vascular bed has a mechanism that prevents expected decrease of blood flow after hemorrhaging and during neurogenic hypertension. The interaction among HAF, PVF, and PVP may be involved in this mechanism.
\end{abstract}

Key words: cerebral ischemia, hepatic arterial flow, portal venous flow, hemorrhage, volume loading.

The hepatic vascular bed has an unusual anatomical arrangement by having input to the sinusoids from two different sources, the hepatic artery and portal vein. This hemodynamic situation is further complicated by the following matter. The evidence of interaction between the events in the two vessels have been reported. A rise and fall in the portal venous pressure causes a decrease and an increase in the hepatic arterial flow (HAF), respectively (SCHENK et al., 1962; LUTZ et al., 1968; LAUTT, 1980). Occlusion of the hepatic artery does not markedly affect the portal venous flow (PVF; EGBERT and RABER, 1952). After a moderate hemorrhage, PVP shows a marked decrease (SelKurt and Brecher, 1956; Greenway et al., 1967b)

Received for publication August 26, 1985 
but the HAF shows ambiguous results: decreases (SELKURT and BRECHER, 1956; GALINDO, 1965; MuNDSCHAU et al., 1966) or increases (SANCETTA, 1953; GreENway et al., 1967b). Concerning the influence of reflexes on hepatic hemodynamics, occlusion of the carotid arteries in cats causes systemic arterial pressure (SAP) rise with little change in HAF (GREENWAY et al., 1967a), and carotid sinus hypotension in dogs increases the HAF and PVF (CARneIro and Donald, 1977).

Studies have been already made on the hemodynamics of the cutaneous, renal, intestinal, and skeletal muscle vascular beds (TAKEUCHI et al., 1979; TAKEUCHI and MIYAKAWA, 1980) and of blood mobilization from the renal, hepatic, jejunal, skeletal-muscle, and hind-paw vascular beds (TAKeUCHI and MiYaKaWA, 1984) during blood pressure oscillation caused by the side pressure exertion procedure (SPEP). Neurogenic constrictions of the superior mesenteric and femoral veins during oscillation were also studied (TAKeuchi and Miyakawa, 1979). These studies have shown how the main peripheral vascular beds, with the exception of a few organs, are involved in the integrative action of the central nervous system.

This study is to investigate the hemodynamics of the hepatic vascular bed during SAP oscillation caused by SPEP in the state of varied circulatory blood volume. We also confirmed the hemodynamical interaction between the two input vessels during SAP oscillation. Circulatory blood volume was varied in order to enhance the interaction. We compared our findings with those from renal, intestinal, and skeletal-muscle vascular beds (TAKEUCHI and MiYaKawA, 1980).

\section{METHODS}

Twenty-seven rabbits of either sex were not fed for $24 \mathrm{~h}$ before the experiment. They were anesthetized with urethane $(0.9 \mathrm{~g} / \mathrm{kg}$, i.m.), paralyzed with gallamine triethiodide $(1.4-2 \mathrm{mg} / \mathrm{kg}$, i.v.), and artificially ventilated. In some rabbits, arterial $\mathrm{pH}, P_{\mathrm{O}_{2}}$ and $P_{\mathrm{CO}_{2}}$ were measured at regular intervals with a blood-gas analyzer (Corning Company, 165), and maintained within normal limits by injecting a sodium bicarbonate buffer solution and adjusting a respirator.

Production of SAP oscillation. The route of blood supply to the brain was surgically restricted to one of the common carotid arteries (MIYAKAwA, 1966a, b; MiyaKaWA et al., 1984). The common carotid artery that was the only remaining route of blood supply to the brain was compressed by side pressure exerted with the device presented in Fig. 1. Stepwise elevation of this pressure caused corresponding rises in SAP superimposed by oscillations. The other common carotid artery was cannulated in order to record SAP.

Side pressure was elevated in $10 \mathrm{mmHg}$ steps up to 90 or $110 \mathrm{mmHg}$, and then to $200 \mathrm{mmHg}$, which completely interrupted blood supply to the brain. Each step was maintained for 1-2 min to achieve a stabilized state.

Measurement of variables in hepatic circulation. The hepatic artery was isolated from the surrounding tissues over the length of 1 to $2 \mathrm{~cm}$ distal to the origin of the gastroduodenal artery. The portion of the portal vein downstream from the 


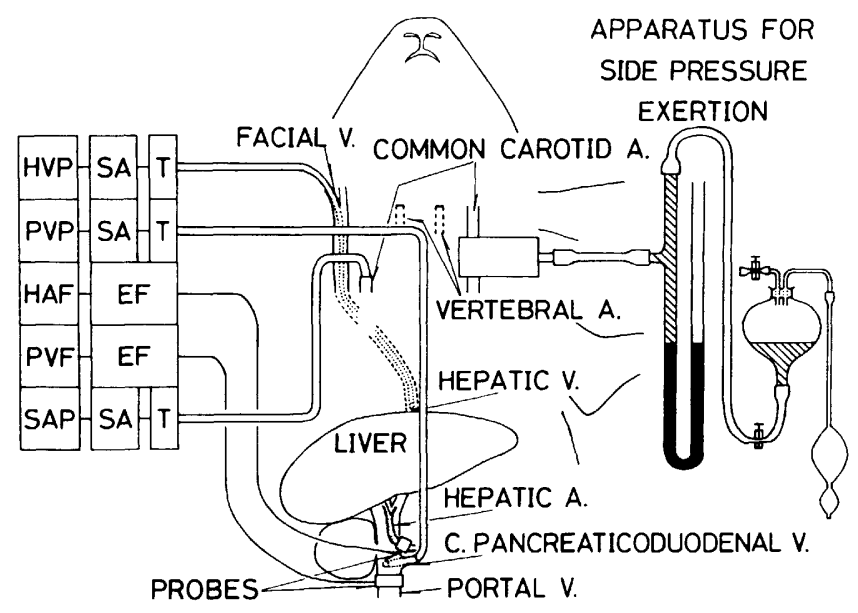

Fig. 1. Schematic representation of the experimental methods. SA, strain amplifier; T, pressure transducer; EF, electromagnetic flowmeter; A, artery; V, vein. HVP, PVP, HAF, PVF, and SAP are described in the text and legend of Fig. 2.

outlet of the cranial pancreaticoduodenal vein was also isolated in the same way. Probes of square-wave electromagnetic flowmeters (Nihon Kohden Co., MFU1200) were placed around these isolated vessels.

The intravascular pressure of the portal and hepatic veins (PVP and HVP) was measured with a strain gauge manometer system that had $2 \mathrm{~s}$ time constant integrators in the circuits and that was connected to the veins with catheters. With the portal vein, the catheter was inserted through the cranial pancreaticoduodenal vein and fixed with its tip in the portal vein. In case of the hepatic vein, a catheter with an inner diameter of $1.2 \mathrm{~mm}$ was introduced into the right facial vein, moved downward, and fixed with its tip being placed in the portion of the hepatic vein within the liver. After the end of each experiment, the exact location of the tip of the catheters was checked. All variables were recorded on a thermal recorder (Nihon Kohden WT-685G). A period of 15 min was allowed between each experiment. The variables of hepatic circulation here are expressed in relation to SAP changes.

We studied the effects of expected catecholamine secretion from the adrenal glands on hepatic hemodynamics during SAP oscillation. Each adrenal gland was isolated from the surrounding tissues, and a snare loop was placed around a bundle of the nerve, artery, and vein. When it was necessary we fastened the snare loops and excluded the glands completely from the circulation.

The animals were weighed before and after the surgery in each experiment. Blood loss due to the operation, usually about $2 \mathrm{ml}$, was compensated by transfusion of fresh blood from another animal.

Changes of circulatory blood volume. Rapid extraction of blood and infusion of Dextran 70-Ringer solution which respectively corresponded to $0.6 \%$ of the 
weight of the animal were done through a catheter which was introduced into the left femoral vein and fixed with its tip in the inferior vena cava. These procedures will be called $0.6 \%$ hemorrhaging and $0.6 \%$ volume loading in this paper. The side pressure exertion experiment was started about $1 \mathrm{~min}$ after the circulatory blood volume was changed.

Analysis of data. Values of SAP, HVP, PVP, HAF, and PVF were measured before and during compression of the common carotid artery, and their means were calculated. When the values were recorded as oscillated or undulated, the means were obtained by levelling off the oscillation or the undulation, which are called the level of oscillation elsewhere (Fig. 2, TAKEUCHI et al., 1979). Statistically significant differences were not found between the means obtained from the cases with and without oscillation or undulation as long as the same magnitude of side pressure was used. The HAF and PVF are expressed in $\mathrm{ml} / \mathrm{min}$ divided by the weight of the animal.

Mean vascular resistance of the hepatic artery (AVR) was calculated by using the following formula:

$$
\mathrm{AVR}=(\mathrm{SAP}-(\mathrm{PVP}-\mathrm{HVP}) / 2) / \mathrm{HAF},
$$

where (PVP-HVP)/2 can be regarded as the sinusoidal pressure (NAKATA et al., 1960).

\section{RESULTS}

\section{$H A F$ and $P V F$}

Normovolemic state (Fig. 2A). The mean HAF (mHAF) increased as SAP rose until the side pressure on the common carotid reached $110 \mathrm{mmHg}$. This was followed by a rapid decrease as SAP rose further. With the initial stepwise elevation of each SAP, the mean PVF (mPVF) had a transient decrease followed by a gradual return to the original level. This progress did not occur at high SAP levels, from 180 to $200 \mathrm{mmHg}$; instead there was a sustained decrease. After restoration of the cerebral blood supply $(0 \mathrm{mmHg}$ of side pressure in Fig. 2) SAP decreased exponentially. During this time, the mHAF decreased slightly more, and then slowly returned to normal, taking about $500 \mathrm{~s}$. The mPVF returned to normal rapidly. The mean HVP ( $\mathrm{mHVP}$ ) and PVP (mPVP) rose slightly with the highest level of SAP.

Effects of $0.6 \%$ hemorrhaging on hepatic circulation (Fig. $2 B$ ). All variables recorded before exerting side pressure decreased after the $0.6 \%$ hemorrhaging. The mHAF increased with the rise in SAP, caused by the SPEP. This was followed by a gradual decrease at the stages where side pressure was higher than $70 \mathrm{mmHg}$.

Effects of $0.6 \%$ volume loading on hepatic circulation (Fig. 2C). The mHVP, mPVP, and mPVF markedly increased after $0.6 \%$ volume loading. The mHAF increase accompanying the SAP rise caused by SPEP was less than those observed under normo- and hypovolemic conditions. This depression can probably be 


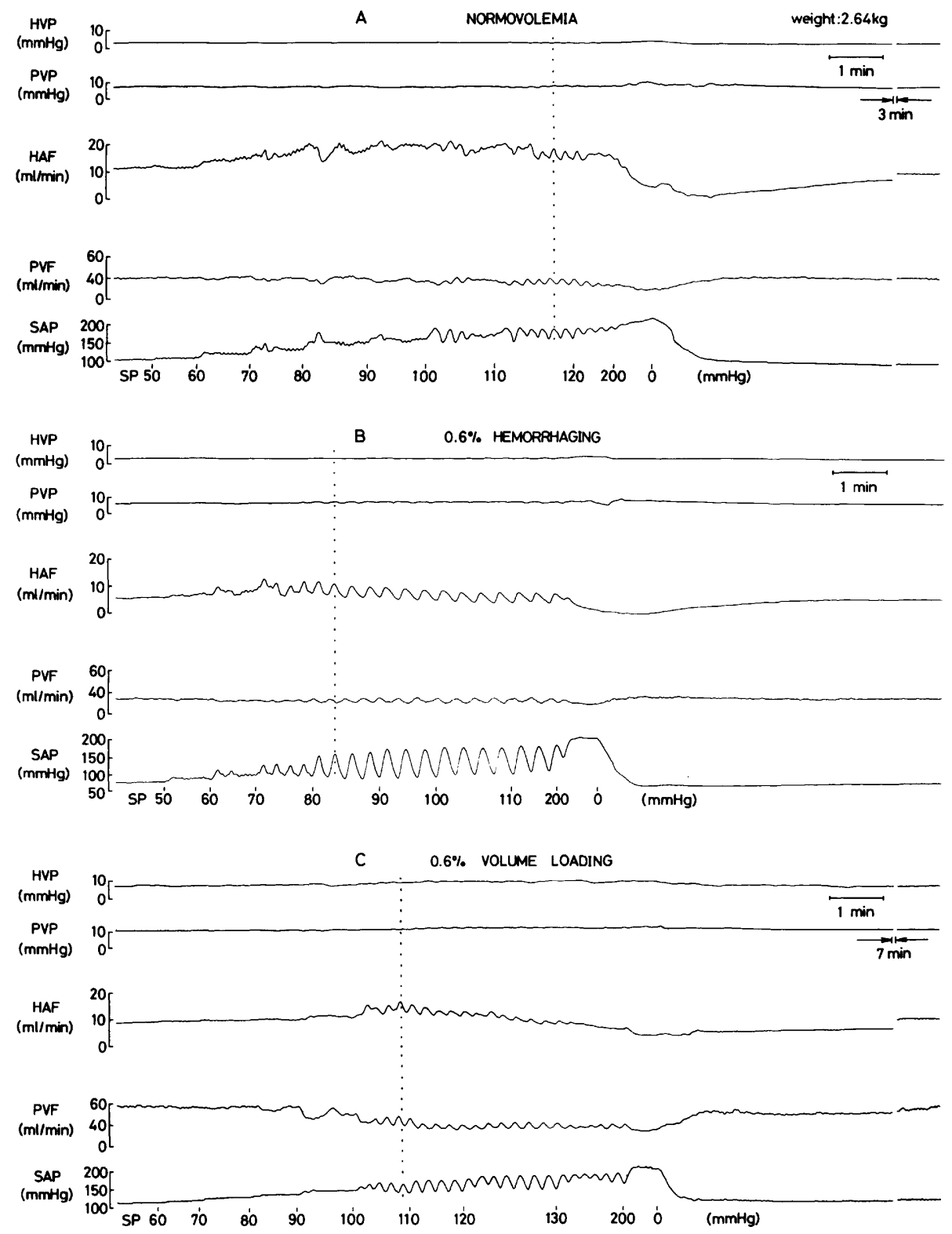

Fig. 2. Records of the hepatic and portal venous pressures (HVP and PVP), hepatic arterial and portal venous flows (HAF and PVF) during systemic arterial pressure (SAP) rise produced by stepwise elevation of side pressure (SP) on the common carotid artery. All data were recorded as mean values. The dotted line in each panel indicates phasic relationships of the curves. 


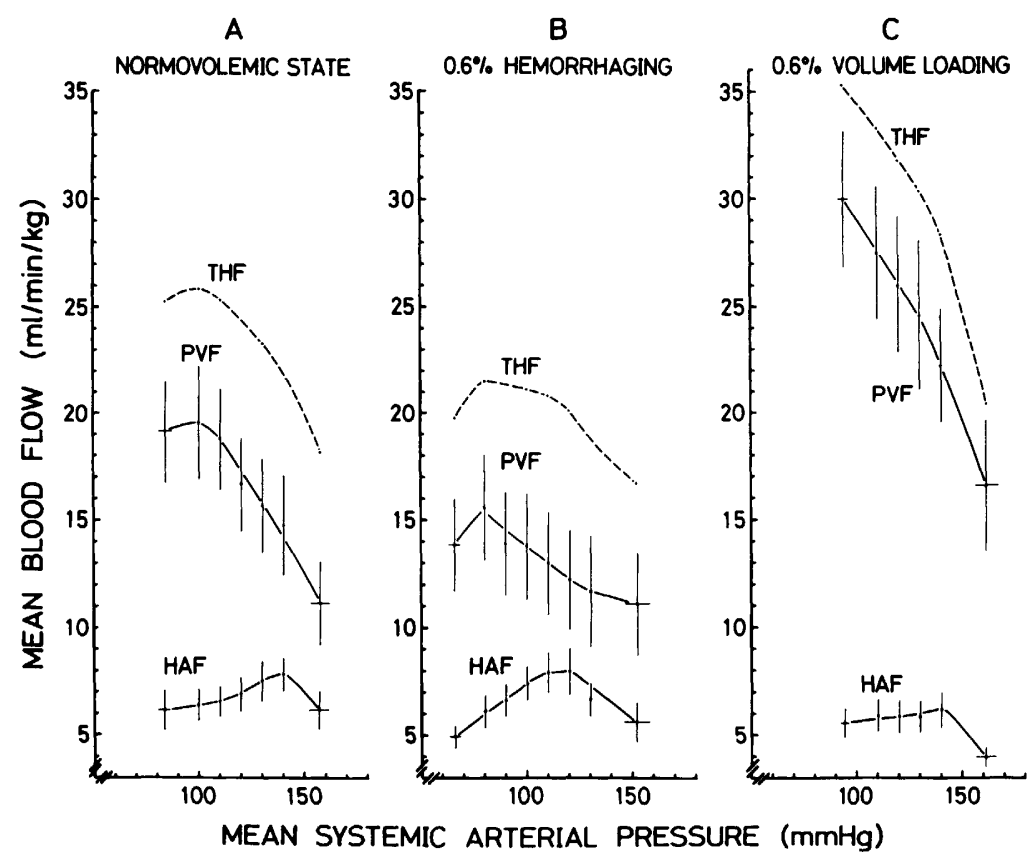

Fig. 3. Relationships of the hepatic arterial, portal venous, and total hepatic (THF) blood flows to mean systemic arterial pressure obtained under normo-, hypo-, and hypervolemic states. THF: see text. Results are shown as mean \pm S.E. $(n=22)$.

ascribed to the elevation of the MPVP and MHVP, which appears almost simultaneously. Thus, the decrease of mHAF accompanying the extreme elevation of SAP caused by exertion of $200 \mathrm{mmHg}$ side pressure was intensified by volume loading.

\section{Relationship between hepatic blood flow and SAP}

The $\mathrm{mPVF}$ and mHAF showed undulatory changes accompanying SAP oscillation during the course of SPEP. All mHAF undulations were in phase with the SAP oscillation except for two occasions, while the mPVF undulations were always out of phase with the SAP oscillation.

Under normovolemic conditions (Fig. 3A). The mPVF decreased almost immediately after an initial slight increase as the SAP was elevated by stepwise increases of side pressure. The $\mathrm{mHAF}$ increased as SAP rose to $140 \mathrm{mmHg}$, and then decreased as SAP rose further.

Effects of $0.6 \%$ hemorrhaging (Fig. $3 \mathrm{~B}$ ). The hemorrhaging caused unanimous decreases in all of the variables recorded. However, when the variables are compared at a particular point of the abscissa, namely at the SAP level of $84.0 \mathrm{mmHg}$, which is control SAP under normovolemic conditions, the mHAF is slightly higher than that under normovolemic conditions. On the other hand the mPVF is much lower than that under normovolemic conditions. In the case of 


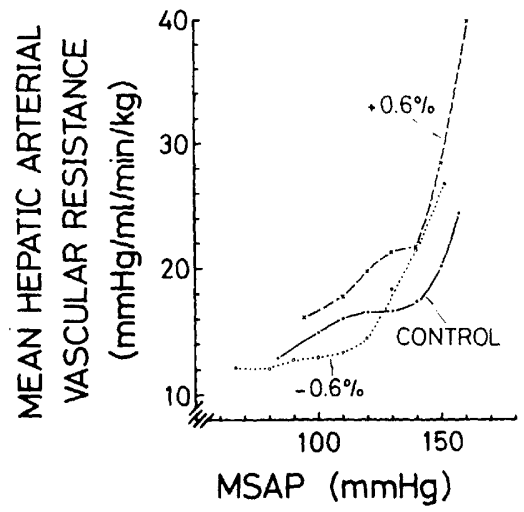

Fig. 4. Relationships of calculated mean vascular resistance of the hepatic artery and mean systemic arterial pressure (MSAP) under varied volemic states.

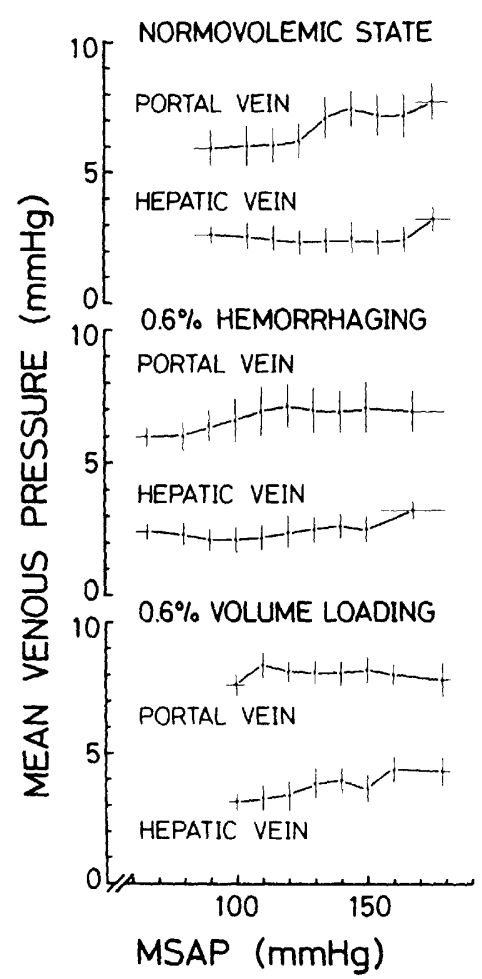

Fig. 5. Relationships of the portal and hepatic venous pressures and the mean systemic arterial pressure obtained under varied volemic states. Results are shown as mean \pm S.E. $(n=8)$. 


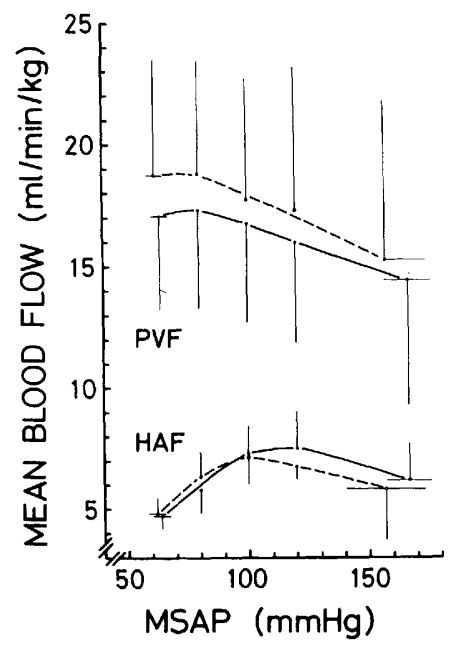

Fig. 6. Effect of functional exclusion of the adrenal glands on hepatic circulatory dynamics during blood pressure oscillation. Dotted and solid lines are results before and after exclusion of the adrenal glands, respectively. Results are shown in mean \pm S.E. $(n=5)$.

hemorrhaging the mPVF decrease and the mHAF increase accompanying the SAP rise were diminished and augmented, respectively. The total hepatic blood flow (THF), the sum of the MPVF and mHAF, was, therefore, well maintained even with moderate SAP rise, as shown in the dashed line in Fig. 3B.

Effects of $0.6 \%$ volume loading (Fig. 3C). Volume loading intensively altered mPVF and mHAF changes during the course of SPEP. Results are shown in relation to the SAP change during this procedure. With volume loading, the mPVF increased 1.5 times and showed a remarkable decrease during the course of SPEP. The increase in mHAF while using SAP elevation was suppressed, which can be ascribed to increased downstream pressure of the MPVP.

Vascular resistance and venous pressure. We calculated the hepatic arterial resistance. Its change during the course of SPEP is shown in relation to SAP changes in Fig. 4. The resistance decreased with hemorrhaging and increased with volume loading.

Under normovolemic conditions, the control PVF (the value before SPEP) was $314 \%$ of the control HAF. Under hypo- and hypervolemic conditions, the former was 279 and $546 \%$, respectively, of the latter.

The mHVP changed proportionally to the circulatory blood volume. The mPVP showed an increase with volume loading but no appreciable decrease with hemorrhaging (Fig. 5). Neither of the mean venous pressures changed significantly during the SPEP. The increase in both MPVP and MHVP produced by the volume loading indicated a rise in sinusoidal pressure that in turn suppressed mHAF elevation as SAP rose, as shown in Fig. 3C. 
Changes after resumption of cerebral blood flow. After resumption of the blood supply to the brain in normovolemia, all variables gradually returned to their original levels. It took $78 \pm 14$ (S.E.), $57 \pm 13$, and $168 \pm 25$ s for SAP, mPVF, and mHAF, respectively. In hypovolemia and hypervolemia, it took $146 \pm 42$ and $101 \pm 16 \mathrm{~s}$ for SAP, $108 \pm 36$ and $128 \pm 30 \mathrm{~s}$ for $\mathrm{mPVF}$, and $264 \pm 46$ and $290 \pm 62 \mathrm{~s}$ for $\mathrm{mHAF}$, respectively. The recovery times for $\mathrm{MHAF}$ in the three volemic states were significantly longer than those for SAP and MPVF.

\section{Effects of functional exclusion of adrenal glands}

Circulatory isolation of the adrenal glands had no clear or significant effects on the hepatic circulatory dynamics during blood pressure oscillation (Fig. 6).

\section{DISCUSSION}

MiYaKaWA et al. (1984) reported that the blood pressure oscillation is mainly due to periodic neurogenic constrictions of the peripheral vascular beds. Therefore, in this condition it is expected that the adrenal medulla may be subjected to the same sympathetic nerve activity and it may secrete catecholamine into the blood stream. It has been reported that epinephrine produces vasodilation of the hepatic artery at lower doses, vasoconstriction at higher doses, and marked portal vasoconstriction (RICHARDSON and WITHRINGTON, 1982). The patterns of changes observed in HAF and PVF with SAP rise caused by SPEP with normovolemia are similar to the effects of epinephrine infusion into both vessels with increasing doses. Thus we examined whether epinephrine plays a role in producing the hepatic hemodynamic changes with SAP elevation that are caused by SPEP. The results indicated no changes in hemodynamic patterns following the functional extirpation of both adrenal glands during blood pressure oscillation. It can be concluded that the effects, if any, of an increase in the catecholamine concentration in the blood on the hepatic circulation were physiologically too weak to be detected.

Carneiro and Donald (1977) reported that in the case of reflex hypertension caused by exposing the carotid sinus to local hypotension in dogs, HAF and PVF increased to 116 and $101.4 \%$ of the control, respectively. In our study, the maximum increase of HAF and PVF were obtained at the later and initial stages of SPEP, respectively. They were 152 and $103 \%$, respectively. These values decreased to 116 and $58 \%$, respectively, as SAP rose further. These differences may be partly attributed to the difference in species, and partly to the difference in the magnitude of activation of the vasomotor nerves between the two experiments. Blood pressure oscillation can produce much stronger activation of the vasomotor nerves than the carotid sinus reflex (Guyton, 1976).

The interaction of HAF and PVP or PVF during blood pressure oscillation is presented in the following matters. It has been already reported that a decrease in pressure or blood flow of the portal vein invariably causes an increase in HAF in anesthetized (COHN and Kountz, 1963; PriCe et al., 1965) and conscious (PrICE et 
al., 1965) dogs. During SPEP, however, we generally obtained a rise in PVP. Therefore, the concurrent HAF increase in our case cannot be ascribed to the reduced PVP or PVF. One factor causing the increase in HAF seems to be a rise in SAP. At the control value in SAP of $84.0 \mathrm{mmHg}$, PVF was markedly decreased but HAF was relatively unchanged by hemorrhaging. This could be caused by a fall in the sinusoidal pressure, namely, the fall of the downstream pressure of the hepatic artery. In the same situation renal blood flow decreases with increasing SAP (TAKEUCHI and Mryakawa, 1980). Here, we found that HAF increased under hypovolemic conditions, and the total blood flow of the liver can be maintained well between 60 to $120 \mathrm{mmHg}$ of mean SAP.

It has been reported that an increase in HVP distended lymphatics and dilated the space of Disse (Brauer et al., 1959). Therefore, HVP increase by volume loading may increase the pressure of the sinusoids and interstitial spaces. This may prevent HAF from increasing during SAP elevation and contribute to the decrease in HAF at the highest SAP level. TAKEUCHI and MiYAKAWA (1980) reported that $0.6 \%$ hemorrhaging increased and $0.6 \%$ volume loading decreased vascular resistance in the renal, intestinal, and skeletal-muscular beds during SAP oscillation. This study gave opposite results in hepatic arterial vascular resistance. This may be attributed to presumed pressure changes in the sinusoids and the interstitial space caused by these procedures. Thus, hemodynamic characteristics of the hepatic vascular bed during blood pressure oscillation became more distinct after hemorrhaging or volume loading, mainly because of the functional dependency among HAP, PVP, and PVF. We found here that hepatic arterial vascular resistance increased to $188 \%$ of the control with the maximum SAP rise caused by exertion of extreme side pressure in normovolemia. This was smaller than the corresponding values for increases in vascular resistance of the renal and superior mesenteric arteries and the abdominal aorta obtained under the same conditions (1030, 574, and 279\%, respectively; TAKEUCHI and MiYaKaWA, 1980). Our results may mean that the hepatic artery is less innervated than the three other vessels.

Stimulation of the hepatic plexus causes little change in PVF but increases PVP (BURTON-OPITZ, 1911, 1913). In contrast, splanchnic nerve stimulation decreases PVF, probably due to splenic and gastrointestinal vasoconstriction (GREEN et al., 1959; SHOEMAKER, 1964). PVF undulations can also be produced by phasic nervous vasoconstriction of the splenic and gastrointestinal beds during SPEP (TAKEUCHI and MiYaKawa, 1980). This could be the reason why PVF undulated out of phase with the SAP wave in this experiment. The change in HAF was in phase with the SAP wave, probably because of the tonic contractions of the hepatic arterial wall.

PVP can be altered by the following factors. First, a decrease in splenic and gastrointestinal blood flow accompanying a SAP rise can reduce it. Second, blood mobilization by contraction of venules and small veins in splenic, hepatic, and gastrointestinal beds, and of large veins such as the superior mesenteric (TAKEUCHI and MiYAKAWA, 1979) and portal veins can increase it. Third, it can be affected by changes in HVP (BRAUER et al., 1959; Hinshaw et al., 1965). PVP was maintained 
at a constant level even after hemorrhaging and tended to increase or remain nearly constant during SPEP. This evidence suggests that graded side pressure exertion causes neurogenic constrictions of the portal venous system.

\section{REFERENCES}

Brauer, R. W., Holloway, R. J., and Leong, G. F. (1959) Changes in liver function and structure due to experimental passive congestion under controlled hepatic vein pressures. Am. J. Physiol., 197: 681-692.

Burton-Opitz, R. (1911) The vascularity of the liver: The magnitude of the portal flow. $Q$. J. Exp. Physiol., 4: 113-125.

Burton-Opitz, R. (1913) The vascularity of the liver: The motor reaction of the portal radicles of the liver. Q. J. Exp. Physiol., 7: 57-74.

Carneiro, J. J. and Donald, D. E. (1977) Change in liver blood flow and blood content in dogs during direct and reflex alteration of hepatic sympathetic nerve activity. Circ. Res., 40: $150-158$.

CoHn, R. and Kountz, S. (1963) Factors influencing control of arterial circulation in the liver of the dog. Am. J. Physiol., 205: 1260-1264.

EGBeRT, H. L. and RABER, R. M. (1952) The physiologic effect of hepatic artery and portal vein ligation: Results of perfusion studies and observations on patients at an operation. Surg. Forum, 2: 153-158.

Galindo, A. (1965) Hepatic circulation and hepatic function during anaesthesia and surgery. Can. Anesthesiol. Soc. J., 12: 262-274.

Green, H. D., Hall, L. S., Sexton, J., and Deal, C. P. (with the assistance of Chakales, H. ) (1959) Autonomic vasomotor responses in the canine hepatic arterial and venous beds. Am. J. Physiol., 196: 196-202.

Greenway, C. V., Lawson, A. E., and Mellander, S. (1967a) The effects of stimulation of the hepatic nerves, infusions of noradrenaline and occlusion of the carotid arteries on liver blood flow in the anaesthetized cat. J. Physiol. (Lond.), 192: 21-41.

Greenway, C. V., Lawson, A. E., and Stark, R. D. (1967b) The effect of haemorrhage on hepatic artery and portal vein flows in the anaesthetized cat. J. Physiol. (Lond.), 193: $375-379$.

Guyton, A. C. (1976) Textbook of Medical Physiology, 5th ed., Saunders Co., Philadelphia, pp. 279-280.

Hinshaw, L. B., Reins, D. A., and Wittmers, L. (1965) Venous-arteriolar response in the canine liver. Proc. Soc. Exp. Biol. Med., 118: 979-982.

LautT, W. W. (1980) Control of hepatic arterial blood flow: Independence from liver metabolic activity. Am. J. Physiol., 239: H559-H564.

Lutz, J., Peiper, U., and Bauereisen, E. (1968) Auftreten und Verhalten venovasomotorischer Reaktionen in der Leberstrombahn. Pfügers Arch., 299: 311-325.

MiYaKAWA, K. (1966a) A method of complete interception of the blood supply to the brain in the rabbit. Med. J. Shinshu Univ., 11: 105-112.

MiYAKAWA, K. (1966b) A method for elicitation of blood pressure oscillation in rabbits. Med. J. Shinshu Univ., 11: 113-125.

Miyakawa, K., Takeuchi, T., Shimizu, T., Hayashi, M., and Kanai, K. (1984) Mechanism of blood pressure waves of the third order. In: Mechanisms of Blood Pressure Waves, ed. by MiYaKawa, K., KoePchen, H. P., and Polosa, C., Japan Scientific Societies Press, Tokyo and Springer Verlag, Berlin, pp. 85-117. 
Mundschau, G. A., Zimmerman, S. W., Gildersleeve, J. W., and Murphy, Q. R. (1966) Hepatic and mesenteric artery resistances after sinoaortic denervation and hemorrhage. Am. J. Physiol., 211: 77-82.

Nakata, K., Leong, G. F., and Brauer, B. W. (1960) Direct measurement of blood pressures in minute vessels of the liver. Am. J. Physiol., 199: 1181-1188.

Price, J. B., Britton, R. C., Peterson, L. M., Reilly, J. W., and Voorhees, A. B. (1965) The validity of chronic hepatic blood flow measurements obtained by the electromagnetic flowmeter. J. Surg. Res., 5: 313-317.

Richardson, P. D. I. and Withrington, P. G. (1982) Physiological regulation of the hepatic circulation. Annu. Rev. Physiol., 44: 57-69.

SANCETTA, S. M. (1953) Dynamic and neurogenic factors determining the hepatic arterial flow after portal occlusion. Circ. Res., 1: 414-418.

Schenk, W. G., McDonald, J. C., McDonald, K., and Drapanas, T. (1962) Direct measurement of hepatic blood flow in surgical patients: With related observations on hepatic flow dynamics in experimental animals. Ann. Surg., 156: 463-471.

Selkurt, E. E. and Brecher, G. A. (1956) Splanchnic hemodynamics and oxygen utilization during hemorrhagic shock in the dog. Circ. Res., 4: 693-704.

Shoemaker, C. P., Jr. (1964) A study of hepatic hemodynamics in the dog. Circ. Res., 15: $216-233$.

Takeuchi, T., Manning, J. W., and Miyakawa, K. (1979) Vascular resistance of intestine, muscle and skin during blood pressure oscillation. Jpn. J. Physiol., 29: 119-130.

TAKeUCHI, T. and MiYaKawa, K. (1979) Neurogenic constriction of the superior mesenteric and femoral veins during systemic blood pressure oscillation in rabbits. Jpn. J. Physiol., 29: 767-780.

TAKEUCHI, T. and MiYAKAWA, K. (1980) Hemodynamic characteristics of regional vascular beds in cerebral ischemic pressor responses. Jpn. J. Physiol., 30: 17-29.

TAKeuCHI, T. and MiYaKaWA, K. (1984) Blood mobilization by regional vascular beds during cerebral ischemic pressor response in rabbits. Jpn. J. Physiol., 34: 469-484. 